\section{" SPONTANEOUS EVOLUTION."}

\section{To the Editor of THE LANCET.}

Sir, - - As the following case relates to an important point in the practice of midwifery, you will perhaps afford space for irs insertion in an early number of your valuable journal.

A few weeks since $I$ was sent for in great haste to attend Mrs. W-n who was then in labour with her second child. Upon making an examination, I found the left hand and forearm protruding through the vagina, and at the os uteri, which was considerably dilated, the scapula and upper part of the back presented. The contractions were so strong that the shoulder and a considerable portion of the back had already been forced through the os uteri. I endeavoured to introduce my hand for the purpose of bringing down the feet, but all my attempts were useless, the uterus was so firmly contracted around the child. Under these circumstances I waited for a short time in hope that the uterus would relax its efforts and enable me to introduce my hand, but instead of the contractions diminishing they were violently increased, the presenting portion of the back revolved upwards, (the hand and forearm still protruding through the vagina), the lower part of the back was forced downwards, and ultimately the breech. By this evolution, I was speedily enabled to accomplish the delivery. The case proves how great is the power of the uterus in rectifying malpositions of the foetus, and that even after the whole upper extremity, and a considerable portion of the trunk, have passed the os uteri, the uterus still retains the power of producing the remarkable phenomenon which is sometimes called "Spontaneous evolution." I am, Sir, your obedient servant,

Stone, Dec. 8th, 1834.

\section{T. B. Barrett.}

SECEECY OF THE EXAMINATIONS OF THE DEAD OR KILLED PATIENTS AT ST. BARTHOLOMEW'S.

To the Editor of The LANCET.-Sir,Allow me first to thank you for the liberal manner in which you have always advocated the cause of the student. 1 am sorry to have again to complain of those frequent sources of grievance at our hospital, the post-mortem examinations. At the commencement of the season, the governors built a new dead-house, and for some time notices of the post-mortems were (regularly posted (thanks to your Journal); but latterly this has gradually been neglected; and for this month past not one notice has been exhibited, although examinations are daily taking place. What is the meaning of this? How are we to learn anatomy and pathology with the present scarcity of subjects and total seclusion from the dead-house? What scandalous breaches of promise are committed by those who pocket our money! How grossly they neglect our interests! Reform is, indeed, wanting at St. Bartholomew's Huspital. You shall hear further on the subject from your obliged servant,

A St. Bartholomew's Student.

St. Bartholomew's Hospital, Dec. 9th, 1834.

\section{ST. BARTHOLOMEW'S HOSPITAL. \\ ULCERATED CANCER OF THE RIGHT BREAST.}

JANE AverY, aged 55, was brought to Faith Ward, Nov. 13, with a large ulcerated cancer of the right breast. The disease commenced seven years ago. At first a small lump only was discovered, which gradually increased, with lancinating pain, and retraction of the nipple. Two years since, her catamenial discharge ceased, and the tumour, after assuming a more active state, became an open ulcer. The patient is much emaciated; her countenance is anxious, and her features have assumed the peculiar sallow and unhealthy appearance which so frequently attends this malady. Pulse werk, appetite impaired; and constant pairs in the axilla and breast, destroy her rest. The ulcer occupies the greater portion of the mamma, and implicates the axillary glands; its surface is very unequal, being in some places deeply excavated, and in others presenting very irregular eminences of a fungoid appearance, standing above the surrounding mass. The edges are much puckered and raised, the discharge is yery considerable during the day, and the foetor is excessive. Cataplasms of bread, saturated with the saturnine lotion, were ordered; but little relief was experienced from their use. The only remedy which appears to lessen her sufferings, is the sedative liquor of opium, diluted with water, and applied over the raw surface, and an opiate pill at night. Since the adoption of this plan, the shooting pains have been less severe, and the nights not so watchful. The patient fancies herself better; but no real sanative influence is evinced by any alteration in the aspect of the breast. The 